sinuent, sinon il faut qu'une cavité quelconque se trouve sur leur passage; elles s'y introduisent alors. Mais beaucoup d'individus doivent périr d'inanition. Nous n'observons que ceux-là seuls qui ont rencontré des conditions dans lesquelles ils puissent vivre.

La sortie de l'imago hors des tiges ne doit rencontrer aucune difficulté essentielle. Évidemment, cet imago ne peut pas plus que sa larve creuser une galerie a travers la paroi dure du C. lanceolatum; mais, la larve étant entrée à la suite d'autres larves, l'imago sortira ḋ la suite d'autres imagos. A cet égard, il n'est pas sans intérêt de remarquer que l'éclosion des insectes parfaits a lieu sensiblement à la mème époque pour Oedemera, Mordellistena et Agromyza. Si l'éclosion des Mordellistena, par exemple, précède celle des Oedemera, ceux-ci attendront simplement quelques jours; dans le cas contraire, les Oedemera trouveront dès leur naissance les voies ouvertes. La concordance s'établira presque nécessairement, quel que soit l'insecte derrière lequel Oedemera s'installe dans une tige, vu que la grande masse des éclosions se produit entre mai et juin. Une coïncidence fâcheuse peut incontestablement se produire, mais tout montre qu'elle se produit rarement.

D'une façon générale, ce qui ressort des observations actuellement connues, c'est que les larves d'Oedemera sont attirées non par telle ou telle autre larve dont elles seraient les commensales habituelles, mais par un certain état du tissu des plantes, que cet état soit déterminé par les agents atmosphériques (1) ou par d'autres animaux.

\title{
Quelques nouveaux Tendipédides [DIPT.] obtenus d'éclosion
}

( 2 e note)

par J.-J. KIEFFER.

Gorynoneura acuticornis, n. sp. - $९$. Brun; abdomen noir, une ligne longitudinale séparant les tergites des sternites, le bord postérieur des tergites, balanciers et pattes blancs, antennes brunes. Yeux ovalaires, non arqués ni amincis en haut, très distants, proba-

(1) Notre collègue A. Chappellier a tout récemment recueilli des branches de chêne, devenues très friables et qui contenaient dẹs larves d' $O$. nobilis à l'exclusion de toute autre larve, 
blement glabres. Palpes de 4 articles graduellement plus ongs. Antennes de 6 articles, dont le $2^{\mathrm{e}}$ et le $3^{\mathrm{e}}$ sont cylindriques et à peine plus longs que gros, $4^{\mathrm{e}}$ et $\mathrm{J}^{\mathrm{e}}$ en ellipse, $6^{\mathrm{e}}$ plus gros que les précédents, composé d'une partie proximale en ellipse, deux fois aussi lon-

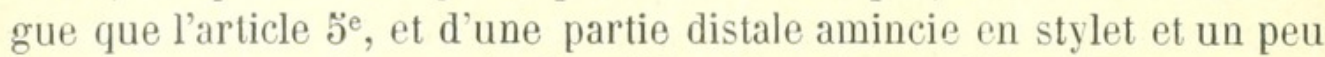
plus courle que la proximale. Ailes blanches, cubitus dépassant le milieu, épaissi, uni au radius, discoïdale sortant en arc du cubitus, bifurcation de la posticale également distante des deux extrémités du cubitus. Tibia postérieur non grossi à l'extrémité, mais muni d'un peigne, $4^{\mathrm{e}}$ article de tous les tarses presque aussi gros que long, un peu plus court que le ${ }^{e}$, crochets tarsaux paraissant bifides, à cause d'une soie grosse et arquée qui semble être un rameau du crochet, empodium atteignant le milieu des crochets. Taille : 1,8 mill. - Larves dans de la mousse.

Reinhardsbrunn.

Bezzia fossicola, n. sp. $-\sigma^{\top} \subsetneq$. Brun noir $\left(\sigma^{\top}\right)$ ou brun clair $(q)$; scutellum et pleures jaunâtres, balanciers blancs, pattes jaunâtres, genoux, tache distale sur les fémurs, anneau situé avant le milieu des tibias et extrémité des tibias bruns, flagellum brun jaune. Articles 3-9 des antennes de la femelle et 3-10 des antennes du mâle subcylindriques, à peine grossis au milieu, d'abord d'un tiers, puis presque deux fois plus longs que gros, à deux appendices subuliformes; panache du mâle remplacé par des soies guère plus longues que chez la femelle et portées par une couronne de granules gros et distants, verticilles de la femelle n'atteignant pas l'extrémité de l'article suivant; articles 11-14 graduellement plus longs chez le mâle, le $11^{\mathrm{e}}$ deux fois et demie aussi

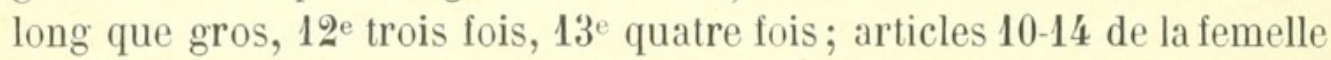
subégaux, cylindriques, ensemble d'un tiers plus longs que les sept précédents réunis, chacun au moins deux fois aussi long que le $\mathbf{9}^{\mathrm{e}}$, sans appendices subuliformes. Mésonotum pubescent. Ailes à soies microscopiques, radius à peine plus court que le cubitus, qui atteint presque le dernier quart de l'aile, la cellule également mince partout, bifurcation de la discoïdale située à peine avant la transversale, bifurcation de la posticale à peine distale de la transversale. Fémur antérieur armé de trois $\left(\sigma^{\top}\right)$ ou de quatre $(q)$ dents noires dans sa moitié distale, tibia avec un éperon et un peigne simple et dense, métatarse sans soies bulbeuses, presque double du $2^{\mathrm{e}}$ article, $4^{\mathrm{e}}$ à peine plus long que gros, cordiforme, 5ొe allongé, plus mince et un peu arqué, crochets simples $\left(\sigma^{\Upsilon}\right)$ ou avec une très petite dent au còté interne près de la base (†); aux 4 pattes postérieures le métatarse est double du 


\section{$2 \mathrm{BHL}$ Biodiversity Heritage Library}

Kieffer, J.-J. 1912. "Quelques nouveaux Tendipédides [Dipt.] obtenus d'éclosion (2e note)." Bulletin de la Société entomologique de France 1912, 101-103.

View This Item Online: https://www.biodiversitylibrary.org/item/36402

Permalink: https://www.biodiversitylibrary.org/partpdf/241304

\section{Holding Institution}

Smithsonian Libraries

\section{Sponsored by}

Smithsonian

\section{Copyright \& Reuse}

Copyright Status: NOT_IN_COPYRIGHT

This document was created from content at the Biodiversity Heritage Library, the world's largest open access digital library for biodiversity literature and archives. Visit BHL at https://www.biodiversitylibrary.org. 